\title{
Daño neurológico en el niño, aspectos clínicos y etiológicos (I)
}

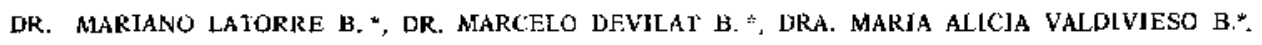

1.- Introducción. Las manifestaciones clínicas de las alteraciones del S. N. C. en los niños, generalmente se agrupan en 6 categorías: neuromotoras, intelectuales, trastornos de la conciencia, neurosensoriales, conductuales y perceptivas, dando origen a cuadros definidos (1).

A objeto de simplificar los conceptos hemos preferido reducir a 5 dichas categorías, llamando

CLASIFICACION FUNCIONAL DE LOS DANOS
NEUROLOGICOS (MODIFICADA) (DENHOFF 196I)

Disfunción Diagn. Clinico Descripción

\begin{tabular}{|c|c|c|}
\hline Neuromotora & Parálisis cerebral & $\begin{array}{l}\text { Incoordinación } \\
\text { neuro muscular } \\
\text { gruesa y fina }\end{array}$ \\
\hline Intelectual & Retardo mental & $\begin{array}{l}\text { Raciocinio y capa- } \\
\text { cidad de } \\
\text { aprendizaje } \\
\text { subnormales }\end{array}$ \\
\hline Conciencia & $\begin{array}{l}\text { Desórdenes convul- } \\
\text { sivos }\end{array}$ & $\begin{array}{l}\text { Alteraciones } \\
\text { electrorrítmicas } \\
\text { corticales } y / 0 \\
\text { subcorticales con } \\
\text { compromiso de } \\
\text { conciencia }\end{array}$ \\
\hline
\end{tabular}

Conductuales Conđucta hiperqui- Distractibilidad fácil nética Concentración dificil

Defectod visuales,

Senso-perceptiva Alteraciones senso- auditivos, aprendiperceptivas zaje y de relación

* Depariamento de Neuralogía. Itosp. Calvo Mackenna.

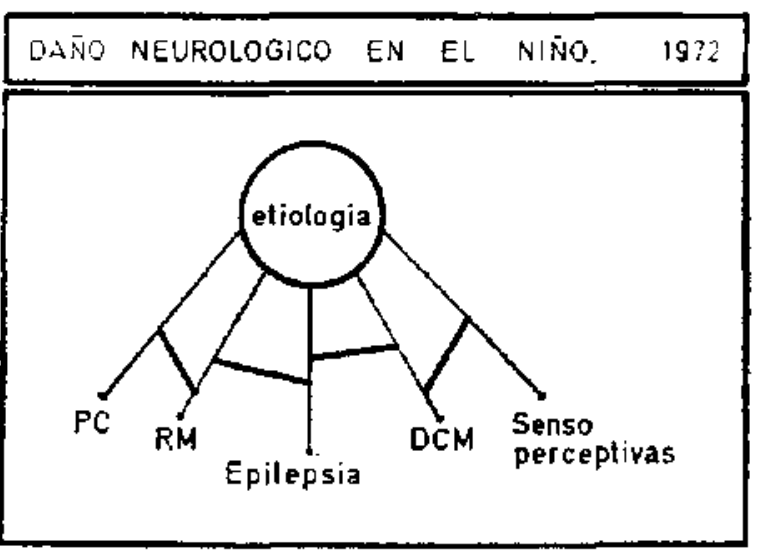

Figura Ne 1

neurosensoperceptivas al conjunto formado por las neurosensoriales y las perceptivas.

En la tabla No 1, modificado de Denhoff, podemos observar que a cada categoría de trastorno corresponde un cuadro clínico determinado. A la disfunción neuromotora corresponde la PC. (Parálisis Cerebral), de la que a priori podemos decir que se trata de una alteración neuromuscular gruesa y fina. Al trastorno intelectual le corresponde el R.M. (Retardo Mental), que compromete el razonamiento y la capacidad de aprendizaje. La alteración de la conciencia significa desórdenes convulsivos. El compromiso de la conducta se mánifiesta como el sindrome hiperquinético, fundamentalmente la D.C.M. (Disfunción Cerebral Mínima) y los trastornos neurosensoperceptivos incluyen toda la patologia que revela dificultades en la percepción del mundo que nos rodea, ya sea por fallas de las estructuras para captarlo, o bien para analizarlo. 
Queremos destacar, (Figura No 1) que estas entidades, etiológicamente, pueden tener causas "similares", que se imbrican entre sí, de tal manera que los mecanismos productores de un trastorпо lo pueden ser de cualquier otro.

Nosotros nos imaginamos que desde una PC. o un R.M., a un leve trastorno de la audición por ejemplo, hay un flujo decreciente de sintomatologia, pero que el fáctor etiológico puede ser el mismo, tanto para los extremos como para las situaciones intermedias.

Aunque ha sido señalado que la duración de la noxa (hipoxia, trauma de parto) o la lesión selectiva de algunas estructuras anatómicas sería la causa que determina el cuadro clínico, se ve con gran frecuencia que éstos no tienen necesariamente un cuadro anátomo patológico característico.

Las alteraciones del $S$. N. C. a que nos hemos referido incluyen un vastísimo campo de la Neurología Infantil, por csta razón aquí comentaremos aquellas que consideramos más frecuentes, desórdenes convulsivos, o menos conocidas (D. C. M.) o más descuidadas (R. M.) o innecesariamente limitantes (Dislexia) o aquellas que siendo muy invalidantes nos plantean dificultades que cstán en nuestras manos resolver (P.C.).

2.- Cuadro clínico. Parálisis Cerebral. Descrita por Little en una monografía clásica en 1861 $(2,3)$. En la tabla $N^{\circ} 2$ mostramos la definición que nosotros usamos en este cuadro, que tienc, como se desprende de ella, características muy variadas.

\section{TABLA N 2 \\ PARALISIS CEREBRAL - DEFINICION HOSP. CALVO MACKENNA}

Grupo de trastomos principalmente motores y no progresivos, ocasionado por compromiso de las estructuras encefálicas durante la etapa de maduración del Sistema Nervioso Central, la que se completa a los 2 años aproximadamente.

Sus características predominantes son en la esfera motora, parálisis, debilidad, movimientos anormales e incoordinación. Suelen agregarse retardo mental, convulsiones, compromiso sensorial y alteraciones síquicas.

Hemos hecho un estudio de 392 niños portadores de PC. en el Instituto de Rehabilitación Infantil de Santiago (I. R. I.) que ingresaron a él para su asistencia y tratamiento en los últimos 15 años (1957-1971). Ellos sólo representan el $28 \%$ de los casos de PC. que fueron evaluados en cl I.R.I. y son sólo una parte de las 3.457 evaluaciones hechas en ese lapso.
En la figura No 2 se muestra la clasificación de la PC. utilizada por nosotros para rotular los casos.

ESOUEMA DE OIAGNOSTICO TOPOGRAFICO PARALISE CBAEBRAL

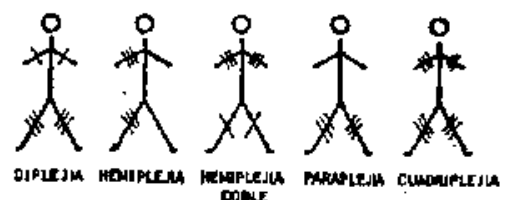

Figura No 2.

En la figura No 3 se observa la forma de prcsentación de nuestros casos desde el punto de vista topográfico, y se destaca que más de la mitad de los casos corresponden a Diplejia y Hemiplejia, lo cual está de acuerdo con otros autores (4). Por ser infrecuentes y de formas muy raras se han excluido del análisis posterior, 1 caso de monoplejia y otro de Diplejia atónica.

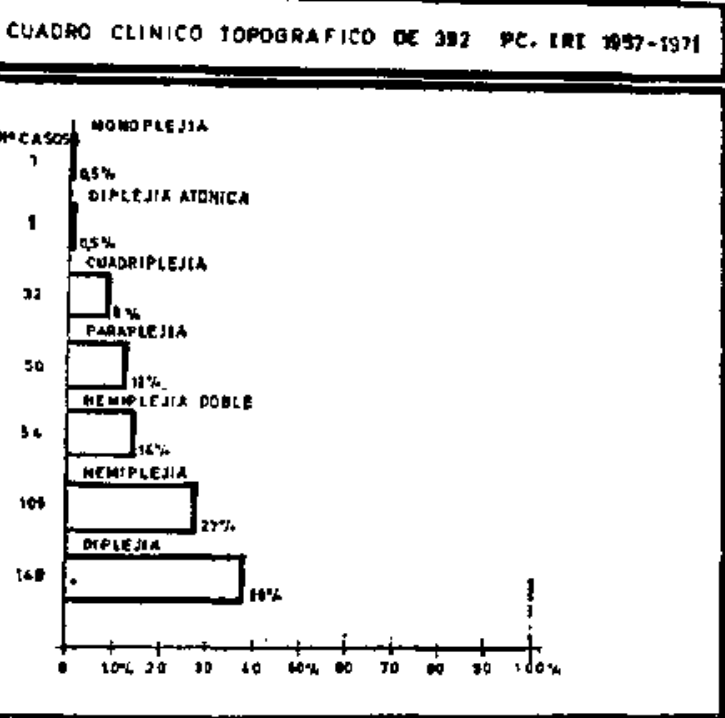

Figura No 3 .

La figura No 4 muestra el tipo de estructura neurológica más comprometida, observândose que al igual que todas las comunicaciones, la forma espástica es la más frecuente, (1-2-3) revelando c] compromiso de la vía piramidal. Las formas atetósicas y atáxicas puras, que corresponden a lesiones extrapiramidales y cerebelosas respectiva- 


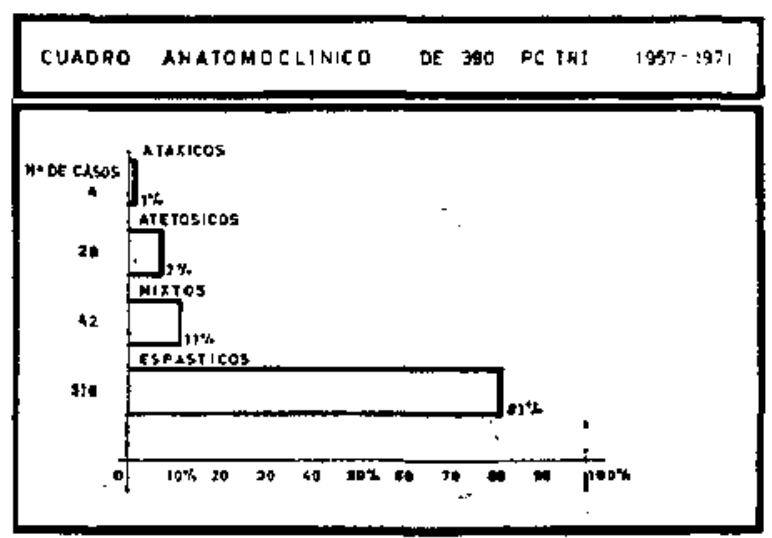

Figurar No 4.

mente, son incluso menos frecuentes que las formas mixtas.

La gravedad de nuestros 390 casos fue analizada de acuerdo al criterio de Rusk (5). Los catalogados como leves son niños sin problemas del lenguaje, caminan sin ayuda de aditamentos y son capaces de satisfacer sus necesidades diarias. El grado moderado corresponde a niños que tjenen problemas de lenguaje, necesitan aditamentos para deambular y tienen dificultades para sus cuidados personales. Siempre necesitan tratamiento. En las formas graves, las características del grupo anterior se maniffestan en forma más intensa y $\mathrm{cl}$ pro. nóstico es más sombrío.

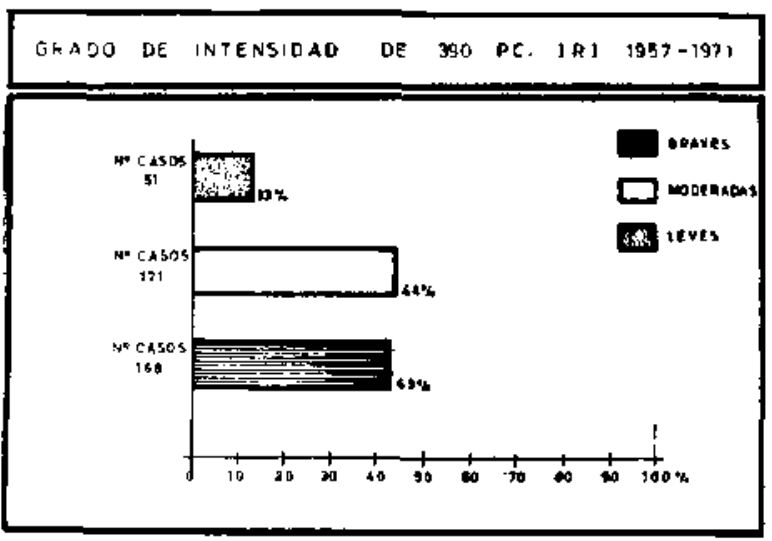

Figura No 5.

En la figura No 5 se observa que el $87 \%$ de los casos tratados en cl I. R. I. corresponden a moderados y graves.

El grado de intensidad, en relación al cuadro topográfico, se observa en la figura $N^{*} 6$ cuyos resultados son en general similares a los obtenidos por otros autores. Hemiplcjia y Paraplejia son más benignás que las Cuadriplejias, Hcmiplejias dobles o Diplejias.

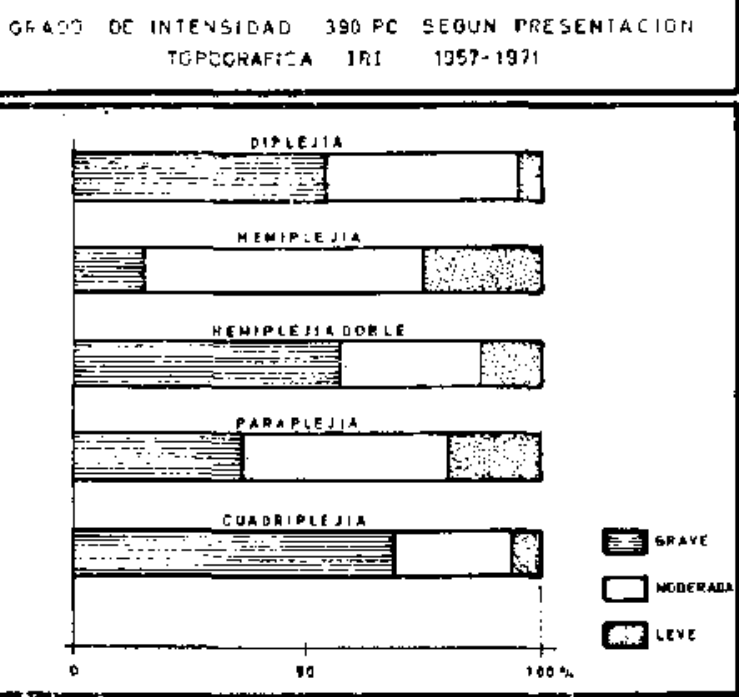

Figura $N \div 6$
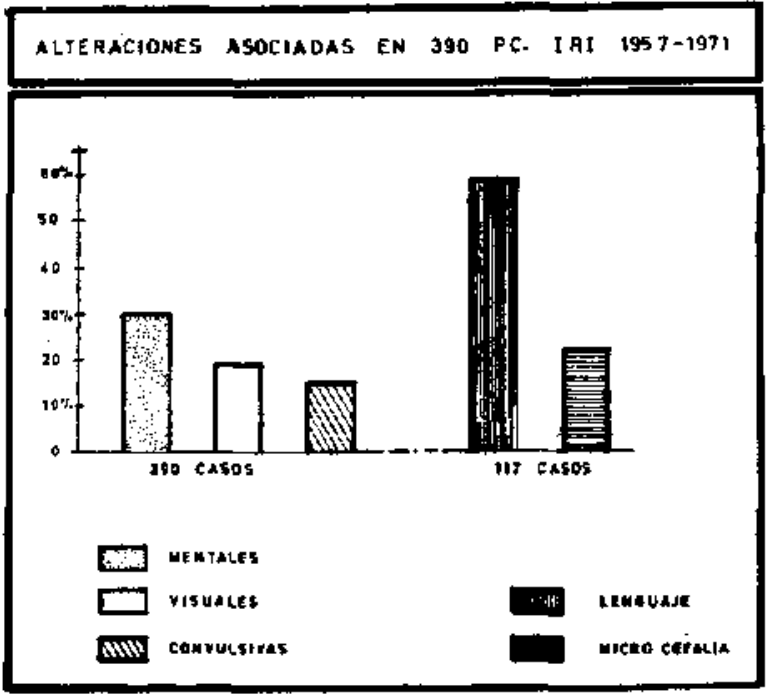

Figura № 7.

La figura No 7 informa acerca de las alteraciones asociadas a la PC. Destacan por su alta frecuencia ef compromiso intelectual y el del lenguaje; menos frecuentes, pero también importantes son el compromiso visual, las convulsiones y la microcefalia.

Epilepsia. La tabla No 3 muestra los clementos de diagnóstico que nosotros utilizamos para rotular los cuadros epilépticos.

En la tablá No 4 hemos detallado nuestra clasificación clínica, en la que destaca la cnorme frecurencia del Grand Mal y dentro de êl, la forma generalizada. El Petit Mal Motor, con sus formas de espasmos mátsivos y crisis aquinéticas, merecen un comentario especial. Los espasmos masivos, 
T A B L A No 3

EPILEFSIA - DLFINICION - HOSPITAL CALVO MACKENNA

Condición caracterizala por episodios frecuentes y transitorios de alteraciones de conciencia o de la experiencia, sea éste seasible o subjetivo y que se acompane o no de movimientos anormales. Estas manifesinciones se Ilaman epilépticas sicmpre que sean producidas por descargas neuronales, bruscits y pastjeras que pueden tener origen focal o ser generalizadas.

\section{TABLA No 4}

CLASIFICACION DE LAS EPILEPSIAS EMPLEADAS EN HOSPYTAL CALVO MACKENNA

I. GRAND MAL 214

$85,6 \%$

a) Generalizado 189

$75,6 \%$

Tónico clónico 159

Tónico 14

Clónico 0

$63,6 \%$

$5,6 \%$

Atónico 16

b) Focal 25
J. Adversivas (lób. Frontal) 2
2. Parietal 2
3. Occipital
4. Temporal
5. Lateralizados 21
$8,4 \%$

II. PETIT MAL (Lapso o ausencia) 13

$5,2 \%$

III. PETIT MAL MOTOR \&

a) Espasmos masivos infantiles 5

b) Crisis Aquinéticas 3

$3,2 \%$

$2 \%$

$1,2 \%$

IV. MIOCLONIAS

a) Sicudidas mioclónicas

b) Epileptia Partialis Continua de Kojewnikaw.

c) Epilepsia miocónica familiar progresiva de Unverricht.

\section{CRISIS PSICOMOTORAS}

(lóbulo tempotal) 9

VI. EQUIVALENTES CONVULSIVOS $16 \ldots$

a) Cefnlea Periódica 5

$6,4 \%$

$2 \%$

b) Trastorno de conducta periódico $5 \ldots$...

c) Crisís viscerales 6

$2 \%$

$2,4 \%$ tambiến llamados Encefalopatía miodónica infantil con hipsarritmia, si bien no representan un volumen importante dentro de las formas de epilepsia, ofrece características que exigen del médico un diagnóstico precoz y un tratamiento eficaz a fin de cvitar secuclas neurosiquicas irrecuperables (6).

Por último, hay dos cuadros clínicos que sin ser epilépticos propiamente tal, se relacionan con ellos y son muy frecuentes. Son las convulsiones febriles y la Apnca emocional. Las primeras, dc etiología aún discutible, aparecen entre los $6 \mathrm{me}$. ses y los 5 años de edad. Su relación con la epilepsia no ha sido demostrada, pero hay estudios que señalan que entre cl 20 a $60 \%$ de los niños que han padecido convulsiones febriles secán más tarde epilépticos.

La Apnea emocional tiene caracteres clínicos que la separan de la epilepsia, pero llama la atención la favorable respuesta al Mysoline que tienc el grado III, de Apnea emocional, forma en que la crisis tiene un período de convulsiones.

Senso perceptivas: Por su frecuencia e importancia, sólo nos vamos a referir a la Dialexia, pues ocuparnos de todas las alteraciones neurosensoperceptivas nos lievaría muy lejos.

La Dislexia se define, (7) (tabla No 5) como una dificultad para lecr, por eso, se presenta en el niño al iniciar su instrucción escolar, y la macstra descubre que el pequeño no sabe las palabras que ya se le enseñaron (7). Es muy importante el diagnóstico preciso de la Dislexia congénita, porque su pronóstico será diferente si se asocia a otras alteraciones del S. N. C. (7). Por otra parte su tratamiento abre las perspectivas que normalmente ticne un niño, a un pequeño que a veces por mal diagnóstico o por no hacerlo puede ser condenado con el rótulo de $\mathrm{R}$. M.

$$
\text { TABLA No } 5
$$

DISLEXIA - DEFINICION - (Critchley)

Enitaña dificultad específica para aprender a leer, a menudo genéticamente determinada, con inteligencia general promedio, sin trastorno emocional ni putología ccrelrral, auditiva, o de la vista concomitantes, ni con deficiencia de la enseñanza convencional. En general la dificultad para leer persiste hasta la edad adulta y se acompaña de dificultad especial para deletrear.

Mediante procedimientos adecuados, se debe scparar entre buenos y malos lectores (7-8) y entre estos últimos, hay que ubicar la Dislexia congénita específica. En términos generales la diferencia de un año entre la edad mental y la edad de lectura (cstimada por el nivel de grado al leer) debe aceptarse como prueba adecuada de 
Dislexia congénita y cuando no hay otros impedimentos que pudieran dificultar al jeer, como son trastornos visuales o auditivos, atención inadecuada o motivación escasa secundaria a factores orgánicos o emocionales (7-8-9).

Entre los aspectos visuales, se han observado movimientos oculares defectuosos durante la lectura de los disléxicos, del orden de tendencia a movimientos regresivos o retrógrados, pequeño número de palabras incluidas en cada desviación del ojo al centro del reglón jnferior, etc. A diferencia de los normales, el disléxico tiene invertido el automatismo de la mirada, y lo hace de derecha a izquierda; todas estas alteraciones son resultado del cuadro en sí y no son su causa (7).

Nos parece importante destacar aquí las conexiones que tiene la distexia congénita específica con la DCM, y señalar sus semejanzas y sus grandes diferencias. Algunos han afirmado que pe queñas lesiones cerebrales, secundarias anexas def parto, pueden causar lo que llamamos dislexia congénita específica. Aseguran que algunos disléxicos tienen signos neurológicos muy leves y pequeñas disrritmias al EEG. Sin embargo, en general, los antecedentes obtenidos en estos niños revelan padres que también han tenido problemas para la lectura y no hay datos acerca de problemas del parto. Por otra parte, es raro encontrar signos neurológicos leves y las alteracjones del EEG. son inconstantes y benignas ( 7 ).

Algunos niños con Dislexia congénita específica, tienen algunos pocos caracteres semejantes (10-11) con DCM., por ejemplo la prueba de repetición de cifras de la escala de Wechsler es inferior al promedio en ambos grupos (pero no en todas las DCM). En ambos, hay trastornos de la lateralidad y mayor frecuencia de dominancia mix$\mathrm{ta}$, cruzada o incompletamente estirblecida. En ambos grupos predominan los varones sobre las niñas. Sin embargo, como se ve en la tabla No 6 (Tomado de Paine) (10) hay muy claras diferencias entre ambos grupos patológicos.

Disfunción cerebral mintima: (DCM). En la tabla Nọ 7 observamos la definición de DCM., aceptada por varios autores (10-11-12) (Tomada de Clements) (11).

El enfoque de la DCM. debe ser realizado desde diversos ángulos, a fin de formarse una idea cabal del niño enfermo. Debe incluir una historia clínica con los antecedentes sobre el embarazo, parto, período de recién nacido; importantes son los datos sobre la madre misma y los que ella y los profesores pueden aportar a la historia de los niños. El examen neurológico y la evaluación sicológica completan los elementos requeridos para efectuar el diagnóstico. (12-13-14).

Existen muchos elementos clínicos para hacer el diagnóstico de DCM., pero con Clements, nos
TABLA N8 6

DIFERENCIAS ENTRE LOS NINOOS QUE PADECEN

DISLEXIA CONGENITA ESPECIFICA Y DISFUNCION CEREBRAL MINIMA *

\begin{tabular}{|c|c|c|}
\hline & $\begin{array}{c}\text { Dislexia congénita } \\
\text { especifica }\end{array}$ & $\begin{array}{l}\text { Disfa } \\
\text { cere }\end{array}$ \\
\hline $\begin{array}{l}\text { Razonamiento abs } \\
\text { tracto y deductivo }\end{array}$ & Bueno & Mato \\
\hline Capacidad aritmética & $\begin{array}{l}\text { Buena, excepto que } \\
\text { no puede leer pro } \\
\text { blemas impresos y } \\
\text { quizás tropiece con } \\
\text { dificultades en cuan. } \\
\text { to a los símbolos } \\
\text { matemáticos }\end{array}$ & Mala \\
\hline
\end{tabular}

Impedimentos per- Principalmente para Múltiples ceptuales palabras

Campo de atención Bueno Limitado

Hiperactividad Poco frecuente, a Frecuente menos que se le im. pulse.

Coordinación visuo. A menudo bueda, mo Mala motora

Escala de Wechsler:

Semejanzas invariablemente

Dibujos de Kohs

Puntuación alta

Inferior a la promedio

C.I. de la escala amplia

Puntuación verbal

en comparación

con puntuación

ide realización

Antecedentes fami- Surelen ser positivos. Suelen ser neliares gativos.

T A B L A NP 7

DISFUNCION CEREBRAL MINIMA - DEFINICION

(Clements)

Niños con inteligencia cercana al promedio o superior a él, que presentan anomalías del aprendizaje, y/o de la conducta, que van de benignos a graves, las cuales guardan relaciones con funciones anormales sutiles del $S$. N. C., debidas a variaciones genéticas, alteraciones bioquímicas, injurias perinatales $\mathrm{u}$ otras enfermedades durante los años de madaración del $\mathrm{S}$. $\mathrm{N}$. y que se caracterizan por varias combinaciones en el déficit de la percepción, conceptualización, lenguaje, memoria, atención, impulsos o función motora.

Síntomas similares pueden complicar a los niños que padecen Parálisis Cerebral, Epilepsia, Retardo Mental, Sordera, Ceguera, a pueden no hacerlo. 
vamos a referir a los 10 más importantes (12-1314-15).

1.- Inteligencia. El test de WISC en su resultado final no está alterado, es normal, sin embargo en general hay déficit en las pruebas de realización más que en las vcrbales.

2.-Déficit perceptuales y motores, que se manifiestan en alteraciones del test de Bender.

3.-Déficit espećficos del aprendizaje.

4.- Déficit de la coordinación general.

5.- Hiperquinesis. Menos frecuentemente hipoquinesis.

6.-- Impulsividad.

7._Labilidad emocional.

8.-- Atención corta y distractibilidad fácil.

9.- Signos neurológicos equívocos y leves. Estrabismo, adiadococinesis, incoordinación de los dedos, alteraciones de la laterabilidad, antecedentes de desarrollo sicomotor y verbal retrasado, etc.

10.- EEG., normal o anormal.

De manera muy general, queremos destacar algunos elementos de la DCM. mediante el análisis de los resultados del trabajo del Dr. Bravo realizado en el Servicio de Psiquiatría del Hospital Calvo Mackenna (14).

En la figura No 8 se muestra la mayor frecuencia de DCM., entre los niños que entre las

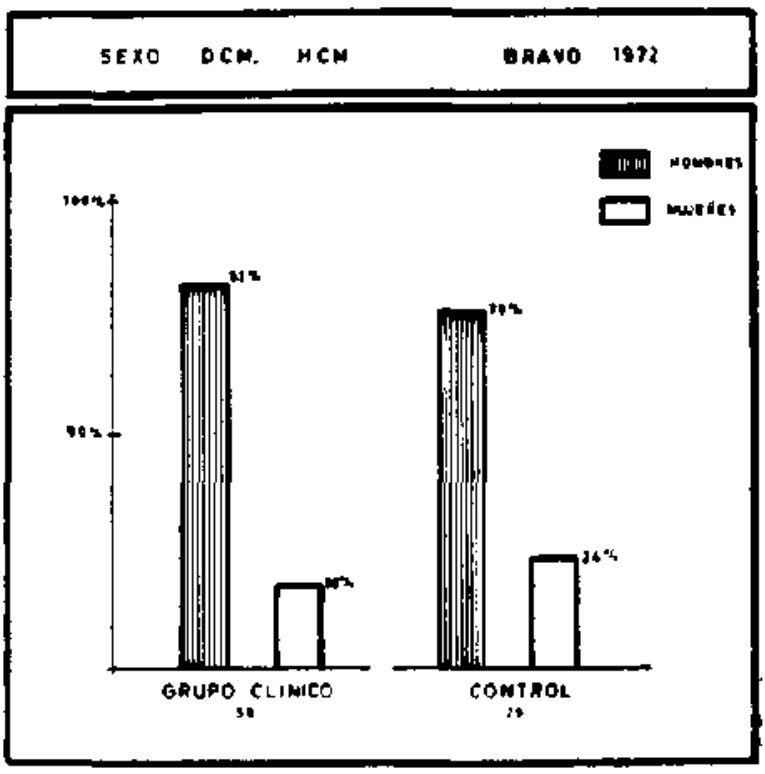

Figura № niñas. En la figura No 9 se muestran tos problemas de aprendizaje, destacándose las dificultades de lectura y escritura.

APRENDIZAJE DCM. HCM. GRAYO 1972

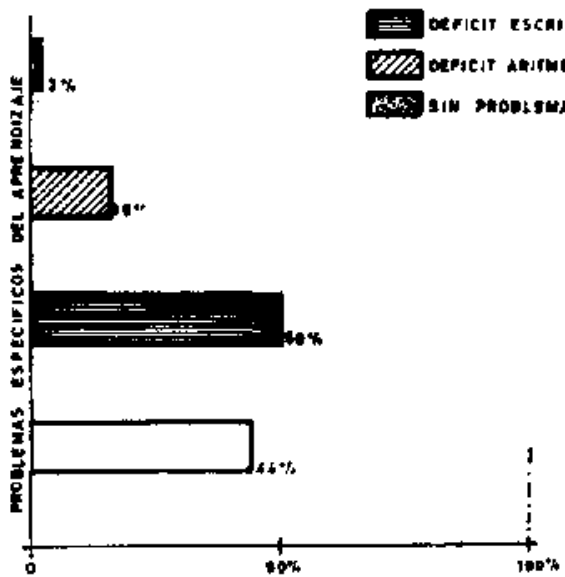

Figura No 9.

En la figura No 10 los problemas de conducta de estos niños y que junto con las alteraciones del aprendizaje, son las causas más frecuentes por las que consultan los padres.

CONDUCIA OCM. HCM BRAVO 1972

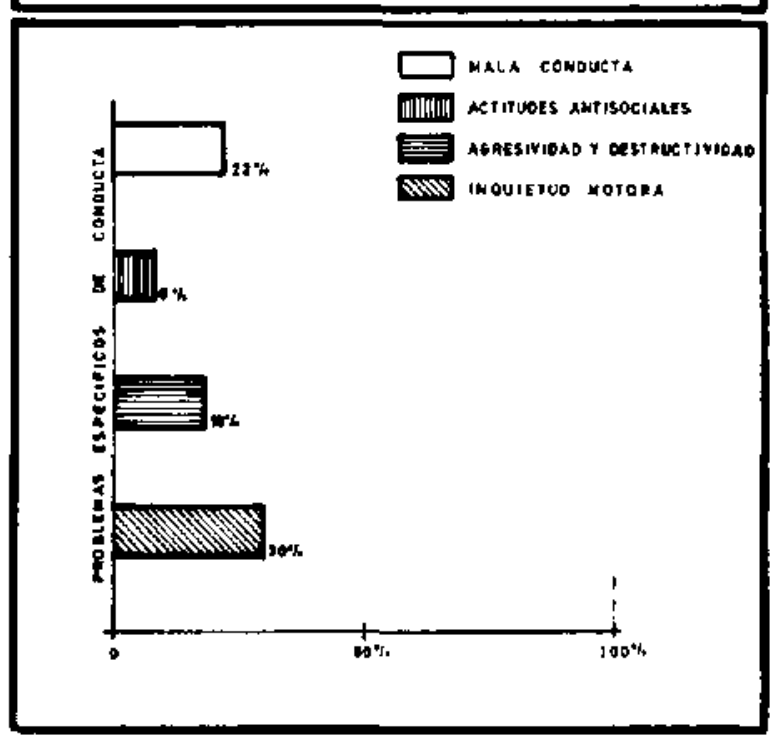

Figura Nミ 10. 
En la figura $\mathrm{N}^{\circ} 11$ destacamos el $\mathrm{Cl}$. de los niños con DMC., comparándoto con el grupo control.

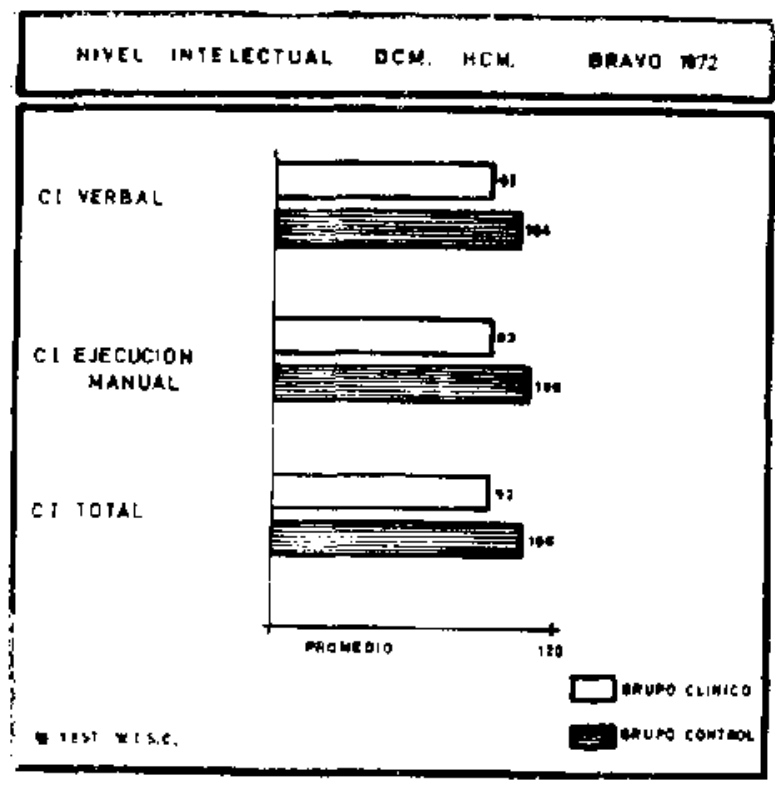

Figura Nu 11.

En la figura $N$ ํ 12 se muestran las aiteraciones, las cuales son característicamente más frecuentes en los enfermos que en los controles. "Las alteraciones emocionales conductuales y de aprendizaje constituyen un trastorno de la personalidad, que sería consecuencia directa de las alteraciones del desarrollo sicomotor y también se-

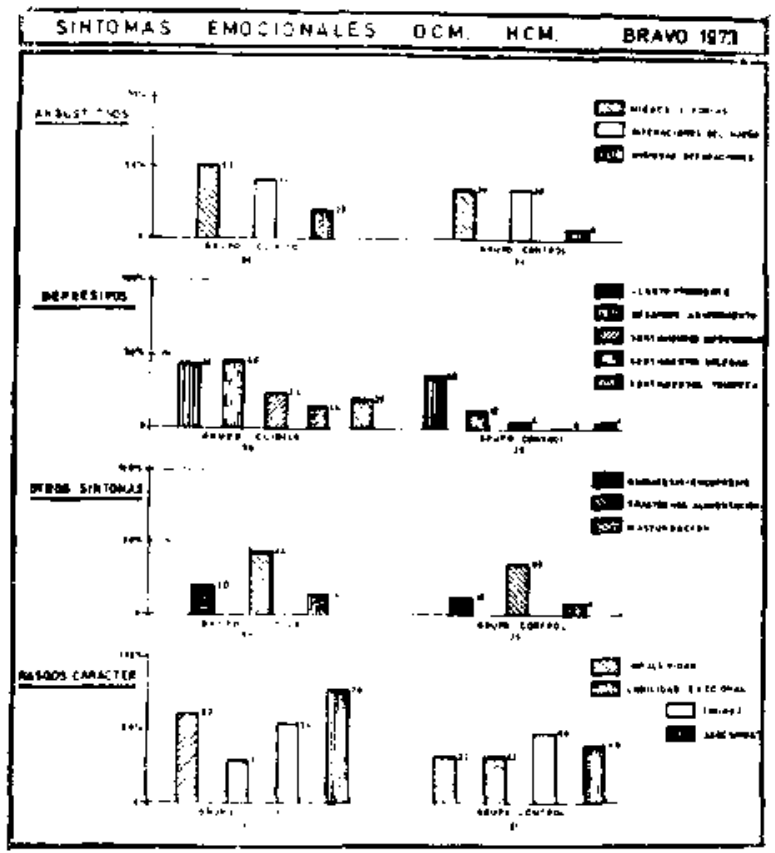

Fisura Na 12. cundario a actitudes inadecuadas del ambiente para las dificultades del autocontrol, tolerancia a frustraciones y aprendizaje escolar" (13).

Retardo mental. Son innumerables los criterios clínicos, sicológicos, conductuales, etc. que se aplican para definir a este cuadro. Por esta razón incluso su denominación es muy variada: retardo mental, retardo sicomotor, deficiencia mental, oligolrenia, amencia, debilidad mental, etc., y sobre ellas hay diversas categorías o grados (16). En consecuencia, una definición de R. M. no será presentada aquí, sino examinaremos los diferentes puntos de vista con que se enfoca generalmente el R. $\mathbf{M}$.

Un criterio sicológico, aceptado por la Academia Americana de Deficiencia Mental, lo define como: "Funcionamiento intelectual general inferior al promedio, que se origina durante el periodo de desarrollo y guarda relación con trastornos de la conducta adaptativa" (17). Característicamente, este es un enfoque evolutivo específico que destaca comparaciones, fundadas en normas adccuadas para la edad cronológica del sujeto. Por otra parte, el enunciado de la definición se hace en términos de inteligencia general, lo cual valora muchos aspectos, como ser, capacidad motora, logros académicos, destrezas de autosuficiencia y sociales, capacidad vocacional y ajuste a la comunidad.

Desde el punto de vista de la educación, se lo define asi: "R. M., como miopía o dureza del oído, es un estado, no es una enfermedad ni siempre es patente. Guarda relación con la forma en que trabaja o no trabaja el cerebro de una persona y la manera en que la capacidad mental del sitjeto es comparable a la de los demás".

Una definición eminentemente médica (Rusk) enfatiza acerca de los problemas físicos: "RM, es princjpalmente, deficiencia mental dependiente de un trastorno del desarrollo cerebral originado antes del nacimiento, durante él o en la primera infancia. EI RM. puede depender de muy diversas enfermedades, accidentes o anomalías genéticas. Puede guardar relación con muchos sindromes $\mathrm{y}$ estados tóxicos al igual que con el medio" (17).

Otro criterio sería el conductual (18). Las personas son retardadas mentalmente, porque se comportan de esta manera, el grado de RM. dependerá que tanto se aparta su conducta de la promedio aceptada como "normal". que apuntan a alteraciones de la capacidad intelectual $y / 0$ de la conducta.

Su estado les acarrea "problemas en su integración social, pues no pueden satisfacer las expectativas que las personas o grupos que las rodean tienen con respecto a ellas" (19).

Existen diversos grados de RM, que se miden por test (W.I.S.C., Binet, Sinan, Terman-Meril, 
etc.). Así, entre 50-70 puntos se habla de RM. ligero; 35-50 RM. moderado; 20-35 RM. severo y 0-20 RM. profundo.

Veremos mấs adelante, que existen otros factores clasificatorios, según otros criterios de enfoque.

En las tablas No 8 y 9 (tomadas de Garrad) (20), queremos destacar cómo la patología aumenta al disminuir el CL y las circunstancias clínicas que son indicaciones para estudios citogenéticos o metabólicos en los niños portadores de R. M..

\section{TABLA Nơ 8}

\section{LA PATOLOGLA AUMENTA AL DISMINUIR EL COCLENTE DE INTELIGENCIA.}

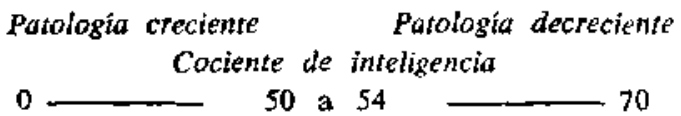

Secuelas graves de enfermedades del sistema nervioso central durante los períodos prenatal, natal o postnatal.

Anomalías citogenéticas

Efectos de genes mayores

Frecuencia de sindromes: de cuando en cuando.
Variabilidad poligénica (ídem)

Factores de experiencia y socioambientales

Secuelas benignas de enfermedad del sistema nervioso central.

Frecuencia de sindromes: raros (variantes benignas: citogenéticas y de genes mayores).

X A B L A No 9

INDICACIONES PARA ESTUDIO CITOGENETICO (Garrad 1968)

- CL $<50-54$

- Tres o más anomalías (cuadro no mendeliano).

- Dermatoglifos anormales, si hay otras dos malformaciones.

-- Cromatina sexual anormal.

\section{INDICACIONES PARA ESTUDIO METABOLICO (Garrad 1968)}

$-\mathrm{CI}<50-54$

- Cuadro semejante en hermano o familiar cercano.

- Signos físicos concomitantes con sindrome conocido.

- Signos neurológicos progresivos o cambiantes.

\section{ETIOLOGIA.}

Parálisis cerebral. Generalmente se acostumbra clasificar las causas de las lesiones neurológicas y con ellas la PC. en prenatal, perinatal y postnatal. En la figura No 13, se observan las más frecuentes causas productoras de las alteraciones, destacándose la prematuridad y los problemas derivados del parto. (Tomado de Lindemann) (4).

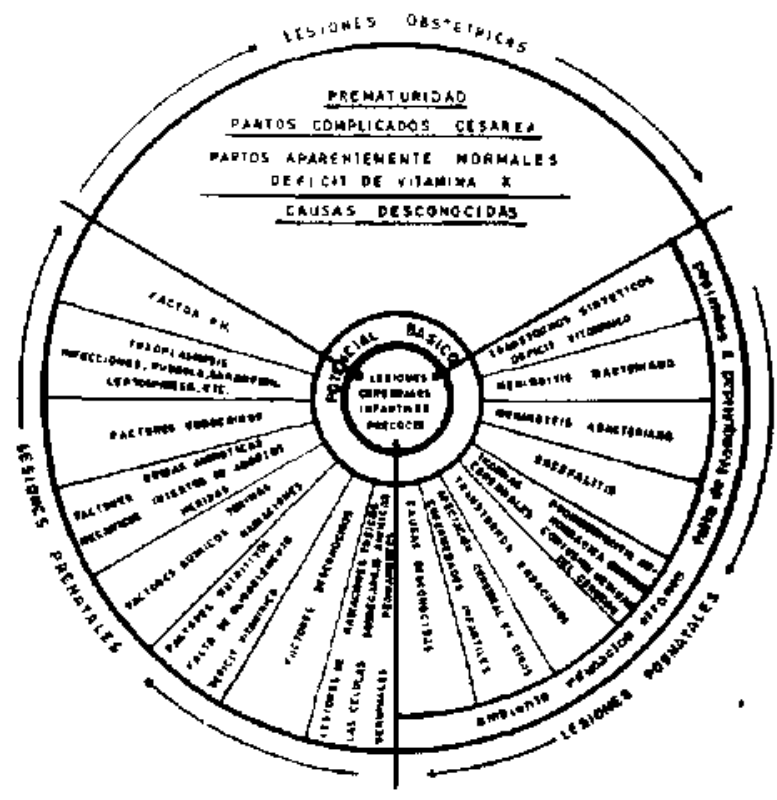

En la tabla No 10 se observa segín varios estudios (1-4-3) la importancia relativa de cada una de las etapas. Destaca aquí, que Derhoff da Ia más alta frecuencia que otros autores para el período prenatal. Rossi y el grupo del Hosp. Calvo $M$. coinciden en sus datos, en tanto que Digennaro y Denhoff plantean algunas diferencias entre ellos. Pensamos que las discrepancias se deberían a nuestro juicio a diferentes procedimientos de investigación y distinta metodología, no standarizada entre los diversos autores.

TA BLA N? 10

PARALISIS CEREBRAL ETIOLOGIA 1972

Rossi Denhoff Digetharo H.C.M.

$\begin{array}{lllll}\text { Prenatal } & 33 \% & 51 \% & 16 \% & 30 \%\end{array}$

Perinatal $55 \% \quad 33 \% \quad 35 \% \quad 55-60 \%$

Postnatal $9 \% \quad 16 \% \quad 45 \% \quad 10-15 \%$

Otras o desconocidas $13 \% \quad 4 \%$ 
En la tabla No 11, queremos enfatizar acerca de la importancia que la hipoxia y la prematuridad tienen como causa de la PC. en 100 casos estudiados en el IRI (21). Otros autores, Erkhardt y Lilienfeld han comunicado que el 23,2 y $22 \%$ de sus casos con PC. eran prematuros (4).

\section{T A B L A No 11}

MMPORTANCIA ETIOLOGICA DE PREMATURIDAD E HIPOXIA EN 10O CASOS IRI. 1969

\begin{tabular}{lllllllllllll}
\hline Prematuridad & $\ldots$ & & $\ldots$ & & & & & & & & & \\
\hline Hipoxia & $\ldots$ & $\ldots$ & $\ldots$ & $\ldots$ & $\ldots$ & $\ldots$ & $\ldots$ & $\ldots$ & $\ldots$ & $\ldots$ & & $37 \%$ \\
\hline
\end{tabular}

Retardo mental. El diagnóstico de este estado, no presenta los elementos objetivos de los otros cuadros aquí tratados. El concepto de RM. es muy variado y distintamente matizado, según los diversos autores como lo veremos más adelante. De ahí también su cofoque etiológico dependerá de cómo cada autor analice eI RM. Por esta razón vamos a exponer aquí diversos criterios para analizar las causas de este cuadro.

En la clasificación etiológica aceptada por la academia Americana de Deficiencia Mental de USA., los factores prenatales son importantemente resaltados. Está basada en el cuociente intelectual (Cl) (1) y Denhoff destaca que "de este gran grupo, el $80 \%$ está clasificado como amencia simple". Son niños cuyos padres tienen deficiencia intelectual, sus familias tienen un trasfondo de deprivación cultural, sicológica y económica y se piensa que este grupo constituye el verdadero tipo de amencia hereditaria. No hay duda que la temprana deprivación puede retardar el desarrollo del niño y esto unido a un ambiente de miedo, ansiedad y agresividad pueden llevar a un permanente daño intelectual que es la amencia simple.

Un segundo grupo de causas que constituyen los "tipos orgánicos o secundarios" tienen una etiología similar a la PC. y los niños afectados pueden tener variados signos neurológicos. Algunos casos tienen causa primaria, como por ejemplo aquellos relacionados con factores genéticos, y otros tienen causas secundarias como son las relacionadas con la hipoxia, infecciones y otros trastornos ocurridos en el período perinatal y postnatal.

En la tabla No 12, mostramos la clasificación de origen siquiátrico (4), en la que se destaca un primer grupo con aquellos factores conocidos o no, que llevan a lesión cerebral. En el $2^{\circ}$ grupo aparecen los factores derivados de deprivación afectiva, sociocultural o económica. En el tercer gcupo, los factores combinados de los grupos anteriores,

En un estudio efectuado en USA. (22) sobre 705 niños cnviados a valorar a una clínica espe-
TABLA Nọ 12

RETARDO MENTAL ETIOLOGIA

(Meneghello 1972)

1. ORIGEN BIOLOGICO factores etiológicos conocidos o desconocidos que llevan a lesión cerebral.

2.- ORIGEN PSICOLOGICO $Y$ SOCIOCULTURAL 3.- ORIGEN BIO-PSICOLOGICO

cializada se demostró que el grupo más frecuente fue la lesión cerebral congénita $19,4 \%$, los problemas derivados de la asfixia, lesión obstétrica mecánica y prematuridad, alcanzaron en conjunto al $10 \%$. Hay que destacar que el $18 \%$ de los niños que fueron enviados por posible RM. resultaron tener inteligencia normal.

Por último, queremos presentar algunas clasificaciones etiológicas del RM., que desde el punto de vista de la genética se han formulado, (tablas No 13 y 14), en los cuales se muestra las enormes

T A B LA No 13

RETARDO MENTAL - ETIOLOGIA DESDE EL PUNTO DE VISTA GENETICO (Wright 1968)

RETARDO MENTAL IDIOPATICO: $85-90 \%$

- Benigno.

- Sin impedimentos ai malformaciones físicas.

- Contribución genética vaga: ¿poligiénicos?

RETARDO MENTAL MEJOR CONOCIDO:

- Grave.

- Hay impedimentos y malformaciones físicas.

- Hay antecedentes de causas externas.

- Contribución genética es posible.

$$
\text { TA B LA Nơ } 14
$$

RETARDO MENTAL - ETIOLOGIA - CLASIFICACION DE SINDROMES (Garrad 1968)

Frecuencin General: $0,3 \%-0,50 \%$ de los escolares CI. $<50-54$

I. SINDROMES CITOGENETICOS: Down E1-DI, etc.

II. SINDROMES DE DEFICIT ENZIMATICO: Fenilcetonuria, Galactosemia, Hemocistinuria, etc.

III. SINDROMES MORFOLOGICOS MACROSCOPICOS O FENOTOPICOS:

n) Sindromes mendelizantes: neurofibromatosis, esclerosis tuberosa y otros.

b) Sindromes no mendelizantes: artiencefalia, sindrome bucal-facial-digital, etc. 
perspectivas que esta rama de la medicina puede ofrecer con el objeto de conocer mejor los cuadros genéticos relacionados con el RM. (20-23).

Epilepsia. Vamos a presentar 2 criterios para analizar la etiología de la Epilepsia. Ford (24), plantea que habría una tendencia constitucional a convulsiones que se manifestaría o no en Epilepsia, si concurren a ella factores de tipo hereditario $y$ de orden precipitante. Figura No 14. En el Hospital Calvo Mackenna luego de estudiar 1.100 casos de Epilepsia, se comprobó que el $85 \%$ de ellos, correspondía a epilepsia esencial (Figura No 15) en la cual no se encontró antecedente de daño neonatal, TBC., inflamación del SNC. y el examen neurológico era normal. Por otra parte, el $15 \%$ demostró antecedentes positivos y fue catalogado como epilepsia secundaria.

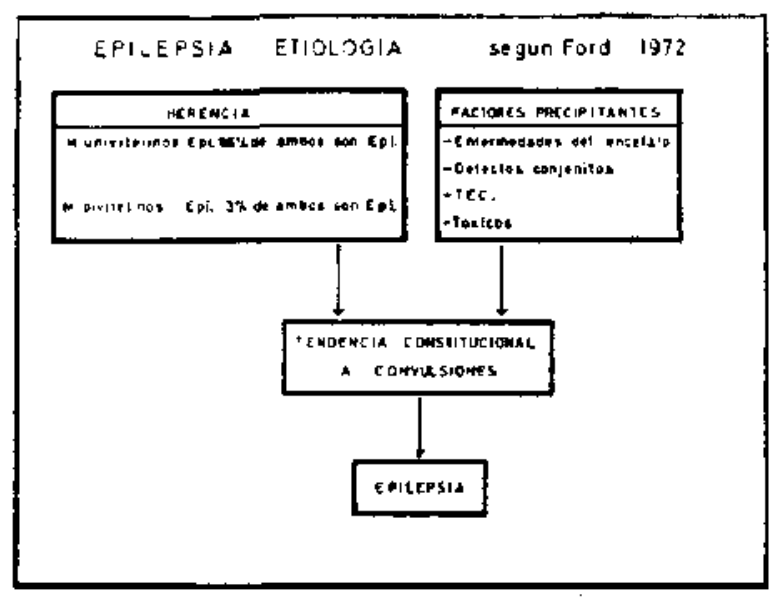

Figura No 14.

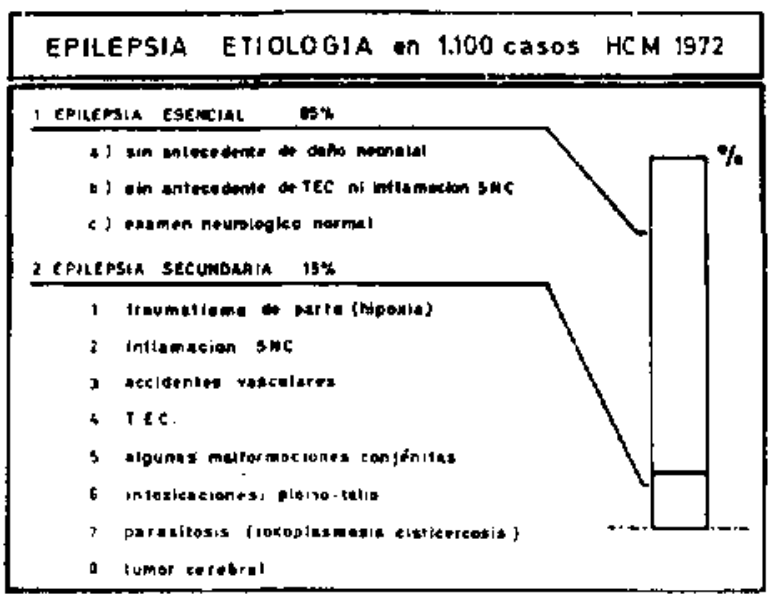

Figura N! 15.

Disfunción cerebral minima. La etiología de la DCM. se mueve entre dos grandes extremos. ¿Es producto de un daño cerebral? o ¿Es sólo un defecto de maduración del SNC?. En la tabla No 15 , se observan diversos porcentajes dados por los autores para explicar las causas de la DCM. Vemos como Bravo (13) postula que un grupo importante de casos tienen antecedentes positivos, $86 \%$, en tanto que para Paine (10), sólo la mitad de los enfermos tendría algún antecedente y la otra mitad no. Para Denhoff (1) habría un grupo sin antecedentes y otro con antecedentes, distribuidos en pre, peri y postnatal. Ambos grupos, sin embargo, tienen jgual comportamiento desde el punto de vista clínico. Algunos casos de DCM. pueden tener asociados RM., PC., Epilepsia y alteraciones neurosensoperceptivas (10, 11), aunque podría discutirse que estos casos estarían englobados dentro del Sindrome hiperquinético más bien que dentro de la DCM. Para el grupo de Olea (15), sólo el 34,9\% tendría anamnesis positiva en cuanto a antecedentes, que son los mismos para la PC. Clements (9), por su parte concluye que hay estudios estadísticos que muestran correlación entre complicaciones del embarazo y DCM.. wí como con enfermedades virales (15) del orden encefalitis por pertussis $y$ sarampión (1). El origen orgánico primario, aún en discusión por la alta proporción de niños sobre niñas que padecen DCM. Se trataría de un desarrollo "normal" en niños rezagados, que serían más susceptibles a los efectos deletéreos del daño cerebral y del stress durante los años críticos del aprendizaje de la conducta simbólica y de la adquisición de los patrones de autoinhibición.

T A B L A Nọ 15

\section{DISFUNCION CEREBRAL MINIMA ETIOLOGIA}

\section{Bravo 1968}

Con antecedentes $86 \%$ (parto distósico hipoxia)

Sin antecedentes $14 \%$

$\begin{array}{ll}\text { Prenatal } & 10-15 \% ? \\ \text { Perinata] } & 70-80 \% \\ \text { Postnatal } & 10-15 \%\end{array}$

Denhoff 1961

Perinata] $\quad 70-80 \%$

Paine 1968

Con antecedentes 50\% (parto distósico, hipoxia, etc.) Sin antecedentes $50 \%$ (alteración maduracion SNC)

Con antecedentes $34,9 \%$

Olea 7968

Sin antecedentes $65,1 \%$

En forma similar, otros autores, Birch, Lauffer y el propio Denhoff (8), han planteado que aceptan los argumentos de organicidad, pero esto no significa necesariamente un daño anatómico cerebral, sino una alteración en el funcionamiento normal del SNC. 
Alteraciones neurosensoperceptivas. Por sil gran variedad sólo nos referiremos a algunas de ellas.

Con respecto a los defectos oculares, sus causas son las mismas de las PC., (1) hipoxia, traumas de parto, infecciones, etc., que lesionan corteza cerebral (lóbulo occipital) cerebro medio, nervios de los músculos oculares, nervio óptico, etcétera.

Son frecuentes los defectos oculares en las PC., que alcanzan, según algunos autores al $50-60 \%$ de los niños afectados (1-4), en tanto que en la población general alcanzan al $3,5 \%$. Similar situación se observa en los trastornos de la audición y lenguaje (4). Cuando hay evidencia de daño cerebral, hay siempre posibilidad de múltiples defectos y entre ellos, este tipo de trastorno es muy frecuente en la PC. $(10-30 \%$ son sordos, en tanto que en la población general, la sordera es de $4 \%$; las alteraciones del lenguaje alcanzan al $94 \%$ de las PC) (1-4).

Con respecto a la dislexia, ésta plantea 3 problemas desde el punto de vista etiológico. Uno, la forma congénita específica, que aparentemente se debe a un retardo en la maduración del SNC, y determinada genéticamente, (10-7), la forma secundaria a efectos del SNC. y sus relaciones con la DCM (7-25) (tabla No 16).

\section{T A B L A No 16}

\section{DISLEXIA - RETARDO LECTOR - ETIOLOGIA (Meneghello 1972)}

\section{Factores sociológicos y psicologicos}

- Defectos cuall y clantitativos en la enseñanza.

- Deficit en estimulación cognocitiva en edad PE.

- Deficiente motivación para aprender:

- por patología social.

- por sicopatolozía personal.

\section{Factores psicofisiológicos}

- Debilidad general.

- Defectos sensoriales.

- Defectos intelectuales.

- Jaqueca cerebral.

- Dislexia congénita específica.

\section{BrBLlOGRAFía}

1.-Denhoff, E. and Pick, 1.: Cerebral Palsy and related disorders. New York. Me. Graw-Hill, 1960.

2.-Wilkins, R. H. and Brody, I. A.: Little's Disease Arch. Neurol. 20: 217, 1969.

3.-Howard, J. y Latorre, M.: Curso de Neurología Infantit, Santiago-Chile. Ed. Universitaria, 1965.
4,-Lindeman, $K .:$ La Parálisis Cerebral. Barcelona. Científico-Médica, 1968.

5.-Rusk, A. H.: Medicina de Rehabilitación. México. Interamericana, 1962.

6.-Devilat, M., Voldivieso, A. Latorre, M., Cox, J., y Mena, F.: Encefalopatía mioclónica infantil con hepsarritmia. Rev. Méd. Chile. Vol. 101, 1973 (En prensa).

7.-Critchley, M.: Dislexia Congénita. Clín. Pediát. 15: $699,1968$.

8.-Meneghello, J.: Pediatría. Buenos Aires, Inter-Médica, 1972.

9.-Brown, J. R., Darley, F. L. y Gómez, M.: Trastornos de la comunicación. Clín. Pediát. 14: 725, 1967.

10.-Paine, R.: Sindrome de "lesión cerebral mínima". Clín. Pediát., 15: 779, 1968.

11.- Clements, S.: National Society for Crippled Childrens and Adults, Chicago, 1964.

12.-Departamento Salud Mental. Apuntes SNS. 1972.

13.-Bravo, L.: Trastornos del desarrollo psicológico en niños con daño cerebrał mínimo. Rev, Chil. Ped. 39: 369, 1968.

14.-Bravo, L.: La sintomatología emocional en la disfunción cerebral mínima. Rev. Chil. Ped. 43: 44, 1972.

I5.-Padilla, A., Olea, R., Pecari, C.J. y George, M.: Nuestra experiencia en Disfunción Cerebral Mínima. Rev. Chil. Ped. 41: 511, 1970.

16.-Warren, S. A.: Valoración psicológica del retardo mental: Revisión de técnicas. Clín. Pediát. 15: 943. 1968.

17.-Cohen, J.: Rehabilitación Vocacional del retardo mental. Clinical Pediát., 15: 1017, 1968.

18.-Johnson, $O$. A.: Educación especial para el retardo mental. Clín. Pediát. 15: 1005, 1968.

19.-Deficiencia mental. Apuntes Servicio de Psiquiatría, Hospital "Luis Calvo Mackenna", SantiagoChile, 1972.

20.-Garrad, $S$. O.: Diagnóstico de Sindrome en el Retardo Mental. Clín. Pediát. 15: 925, 1968.

21.-Ancelovici, s.: Parálisis Cerebral Infantil. Contribución a sa estudio. Tesis de título U. de Chile, 1970.

22.- Pearson, P.: Papel del módico en el diagnóstico y la asistencia de retardos mentales. Clín. Pediát. 15: 835, 1968.

23.-Wright, 5. W. and Sparkes, R. S.: Orientación Genética en el retardo mental. Clín. Pediát. 15: 905, 1968.

24.-Ford, F.: Enfermedades del Sistema Nervioso. Rosario (Rep, Argentina). La Médica, 1957.

25. - Ingram. T.: Trastornos del lenguaje en la infancia. Clín. Pediat. 15: 611, 1968. 